\title{
METRIC AND SYMMETRIC SPACES
}

\author{
PETER W. HARLEY III
}

\begin{abstract}
In this paper we give an alternative proof, without reference to Urysohn's lemma, of the metrization theorem of Bing [2], Nagata [6], and Smirnov [8] via the theory of symmetric spaces as developed by $\mathbf{H}$. Martin in [5].
\end{abstract}

A symmetric $d$ on a point set $X$ is a function $X \times X \rightarrow[0, \infty)$ satisfying (1) $d(x, y)=0$ if and only if $x=y$, and (2) $d(x, y)=d(y, x)$. A topology $T$ on $X$ is said to be determined by $d$ provided that for every subset $U$ of $X$, $U$ belongs to $T$ if and only if it contains an $\varepsilon$-sphere $N(p ; \varepsilon)(=\{x: d(p, x)<$ $\varepsilon\})$ about each of its points $p$. The data $X, d$, and $T$ is called a symmetric space. Such a space need not be Hausdorff or first countable, but H. W. Martin [5] has proved the theorem below.

THEOREM 1. Let X be a topological space symmetrizable via a symmetric d. If $d(K, F)>0$ whenever $K \cap F=\varnothing, K$ is compact, and $F$ closed, then $X$ is metrizable.

This theorem strengthened an earlier theorem of A. V. Arhangel'skir [1], who introduced the notion of symmetric spaces. Martin achieves a proof of Theorem 1 by showing that $X$ must satisfy the hypotheses of Mrs. Frink's theorem [3], a classical result in metrization theory. As a corollary of Theorem 1, Martin (and Arhangel'skiī) obtains the theorem of S. Hanai and K. Morita [4], and A. H. Stone [9] on the metrizability of perfect images of metric spaces.

The purpose of this paper is to obtain the metrization theorem of Bing [2], Nagata [6], and Smirnov [8] as a consequence of Theorem 1. It is interesting to note that Urysohn's lemma is never used in this approach, as was the case in the approach used by D. Rolfsen in [7]. More specifically, let us assume that $X$ is a regular, $T_{1}$ space with a $\sigma$-locally finite base $\mathscr{B}=\bigcup_{n=1}^{\infty} \mathscr{B}_{n}$, where $\mathscr{B}_{n}$ is locally finite and $\mathscr{B}_{n} \subset \mathscr{B}_{n+1}, n \geqq 1$.

Received by the editors May 8, 1973 and, in revised form, August 10, 1973.

AMS (MOS) subject classifications (1970). Primary 54E35, 54E25; Secondary 54D10, 54D20.

Key words and phrases. Metrizable space, symmetrizable space, $\sigma$-locally finite base.

(c) American Mathematical Society 1974 
For $x, y \in X, x \neq y$, put $m(x, y)=\min \left\{n: \exists B \in \mathscr{B}_{n}\right.$ with $\left.x \in B, y \notin \bar{B}\right\}$, $t(x, y)=1 / m(x, y)$, and $d(x, y)=\max \{t(x, y), t(y, x)\}$. Also, put $d(x, x)=$ 0 . Then we shall prove the following theorem.

THEOREM 2. $X$ is symmetrizable via $d$. Furthermore, $d(K, F)>0$ whenever $K \cap F=\varnothing, K$ is compact, and $F$ closed. Therefore, $X$ is metrizable.

Proof. Denote by $T$ and $T_{d}$ the given and $d$-induced topologies on $X$, respectively. We must show that (1) $T \subset T_{d}$, (2) $T_{d} \subset T$, and (3) $d(K, F)>$ 0 whenever $K \cap F=\varnothing, K$ is compact, and $F$ closed.

To establish (1), assume that $B \in \mathscr{B}, x \in B$. Choose $B_{1} \in \mathscr{B}$ such that $x \in B_{1} \subset \bar{B}_{1} \subset B$. If $B_{1} \in \mathscr{B}_{n}$, we have $N(x ; 1 / n) \subset \bar{B}_{1} \subset B$, so that $B$ is open in $T_{d}$.

To establish (2), let $F$ be a $T_{d}$-closed set. If $F$ is not $T$-closed ( $X$ is first countable because of $\sigma$-locally finite $\mathscr{B}$ ), there is a point $x \notin F$ and a sequence $x_{1}, x_{2}, \cdots$ of points in $F$ converging to $x$. We shall show that

(i) $\lim _{i \rightarrow \infty} t\left(x, x_{i}\right)=0$,

(ii) $\inf \left\{t\left(x_{i}, x\right): i \geqq 1\right\}=0$, so that

(iii) $\inf \left\{d\left(x, x_{i}\right): i \geqq 1\right\}=0$ holds, which contradicts $d(x, F)>0$.

To this end, let $x \in B \in \mathscr{B}_{n}$. Denote by $U$ the intersection of all members of $\mathscr{B}_{n}$ containing $x$. There exists a positive integer $N$ satisfying $x_{i} \in U$ for $i \geqq N$, whence $t\left(x, x_{i}\right)<1 / n$. Since $n$ can be chosen as large as we please, (i) follows.

As for (ii), let $x \in B \in \mathscr{B}_{n}$. Denote by $V$ an open neighborhood of $x$ that intersects only finitely many members of $\mathscr{B}_{n}$ and satisfies $V \subset B$. Choose $N$ so that $x_{i} \in V$ for $i \geqq N$. Whenever $i \geqq N$, let $U_{i}$ represent the intersection of all members of $\mathscr{B}_{n}$ containing $x_{i}$. It follows that for infinitely many such values of $i$, the sets $U_{i}$ are identical, there being only finitely many such intersections. Denoting such a common value by $U$, it is clear that $x \bar{\in} \bar{U}$, and therefore that $t\left(x_{i}, x\right)<1 / n$. This establishes (ii), (iii), and (2).

To establish (3), let $K$ be compact, $F$ closed, and $K \cap F=\varnothing$. Let $B_{1}, B_{2}, \cdots, B_{k}$ be a finite cover of $K$ by members of $\mathscr{B}$ with $\bar{B}_{i} \cap F=\varnothing$, $i=1, \cdots, k$. Choose $n$ such that $B_{i} \in \mathscr{B}_{n}, i=1, \cdots, k$. Then we have $0<1 / n \leqq t(K, F) \leqq d(K, F)$.

\section{REFERENCES}

1. A. V. Arhangel'skiǐ, Mappings and spaces, Uspehi Mat. Nauk 21 (1966), no. 4 (130), 133-184=Russian Math. Surveys 21 (1966), no. 4, 115-162. MR 37 \#3534.

2. R. H. Bing, Metrization of topological spaces, Canad. J. Math. 3 (1951), 175-186. MR 13, 264.

3. A. H. Frink, Distance functions and the metrization problem, Bull. Amer. Math. Soc. 43 (1937), 133-142.

4. S. Hanai and K. Morita, Closed mappings and metric spaces, Proc. Japan. Acad. 32 (1956), 10-14. MR 19, 299. 
5. H. W. Martin, Metrization of symmetric spaces and regular maps, Proc. Amer. Math. Soc. 35 (1972), 269-274.

6. J. Nagata, On a necessary and sufficient condition for metrizability, J. Inst. Polytech. Osaka City Univ. Ser. A Math. 1 (1950), 93-100. MR 13, 264.

7. D. Rolfsen, Alternative metrization proofs, Canad. J. Math. 18 (1966), 750-757. MR 33 \#6585.

8. Ju. M. Smirnov, A necessary and sufficient condition for metrizability of a topological space, Dokl. Akad. Nauk SSSR (N.S.) 77 (1951), 197-200. (Russian) MR 12, 845.

9. A. H. Stone, Metrizability of decomposition spaces, Proc. Amer. Math. Soc. 7 (1956), 690-700. MR 19, 299.

Department of Mathematics, University of South Carolina, Columbia, South Carolina 29208 